\title{
Comprehensive Analysis of the Expression, Relationship to Immune Infiltration and Prognosis of TIM-1 in Cancer
}

\author{
Xiaoxiao Kong ${ }^{1}$, Meili $\mathrm{Fu}^{2}$, Xing $\mathrm{Niu}^{3}$ and Hongxing Jiang ${ }^{1 *}$ \\ ${ }^{1}$ Department of General Surgery, Linyi People's Hospital Affiliated to Shandong University, Linyi, China, ${ }^{2}$ Department of \\ Infectious Diseases, Linyi People's Hospital Affiliated to Shandong University, Linyi, China, ${ }^{3}$ Department of Second Clinical \\ College, Shengjing Hospital Affiliated to China Medical University, Shenyang, China
}

OPEN ACCESS

Edited by:

Samuel J. Klempner, Massachusetts General Hospital

Cancer Center, United States

Reviewed by: Gang Sun,

People's Liberation Army General

Hospital, China Jichang $\mathrm{Hu}$,

Renmin Hospital of Wuhan

University, China

*Correspondence:

Hongxing Jiang

hongxing4119@163.com

Specialty section

This article was submitted to Gastrointestinal Cancers,

a section of the journal

Frontiers in Oncology

Received: 04 February 2020

Accepted: 01 June 2020

Published: 04 September 2020

Citation:

Kong X, Fu M, Niu X and Jiang H (2020) Comprehensive Analysis of the Expression, Relationship to Immune Infiltration and Prognosis of TIM-1 in

Cancer. Front. Oncol. 10:1086. doi: 10.3389/fonc.2020.01086
TIM-1 is a critical gene that regulates T-helper cell development. However, little research has revealed the distribution, prognosis, and immune infiltration of TIM-1 in cancers. TCGA, GEO, Oncomine, TIMER, Kaplan-Meier, PrognoScan, GEPIA, TISIDB, and HPA databases were used to analyze TIM-1 in cancers. High TIM-1 expression was observed in bladder, cholangio, head and neck, colorectal, gastric, kidney, liver, lung adenocarcinoma, skin, uterine corpus endometrial, and pancreatic cancers compared to the normal tissues, and immunofluorescence shows that TIM-1 is mainly localized in vesicles. Simultaneously, high TIM-1 expression was closely related with poorer overall survival in gastric, lung adenocarcinoma, and poorer disease-specific survival in gastric cancer in the TCGA cohort, and was validated in the GEO cohort. Moreover, high expression of TIM-1, correlated with clinical relevance of gastric cancer and lung adenocarcinoma, was associated with tumor-infiltrating lymphocytes in lung adenocarcinoma and gastric cancer. Finally, immunohistochemistry showed TIM-1 expression was higher in lung adenocarcinoma and gastric cancer compared to the normal tissues. In summary, we applied integrated bioinformatics approaches to suggest that TIM-1 can be used as a prognostic biomarker in gastric and lung adenocarcinoma, which might provide a novel direction to explore the pathogenesis of gastric and lung adenocarcinoma.

Keywords: cancer, TIM-1, bioinformatics, immune infiltration, biomarker

\section{INTRODUCTION}

Cancer is the second leading cause of death worldwide, and the treatment of cancer is still based on traditional surgery, radiotherapy, and chemotherapy $(1,2)$. With further research done on the molecular mechanism of tumorigenesis and development, research on targeted molecular therapy has made great progress. However, due to the high heterogeneity of tumors, new treatment methods are urgently needed (3). Immunotherapy with immune checkpoint blocking, tumor infiltrating lymphocytes, chimeric antigen receptor $\mathrm{T}$ cells, and $\mathrm{T}$ cell receptor chimeric $\mathrm{T}$ cell have achieved certain effects in the treatment of various tumors. However, only $10-20 \%$ of the population can benefit $(4,5)$. Due to the heterogeneity of tumors, the current biomarkers for predicting prognosis have certain limitations. Therefore, this field requires new biomarkers as prognostic indicators to effectively enhance prognosis and individualized treatment. 
TIM protein is a kind of transmembrane glycoprotein expressed on the surface of T cells with similar structural motifs. The human TIM gene family is located in chromatin 5q33.2, and includes TIM- 1, TIM- 3, and TIM-4 (6). TIM-1 protein was first discovered as a receptor of hepatitis A virus in kidney cells of African green monkeys, and plays a role in T-helper cell development (7). TIM-1 is expressed in CD4+ T cells and starts transcription at the initial stage of antigen stimulation, which provides a costimulatory signal for $\mathrm{T}$ cell activation, participates in $\mathrm{T}$ cell proliferation and differentiation, and inhibits the occurrence of peripheral tolerance (8-10). These findings suggest TIM-1 is a key gene that can regulate T cells and is likely to be an immune marker in cancer.

This study was to analyze the expression and prognosis for TIM-1 and relevance for immune infiltration in cancer. Firstly, we detected the distribution and expression of TIM-1 in human cancer. Secondly, we comprehensively analyzed TIM1 correlation with prognosis of cancer, which was validated in GEO database. Moreover, we detected the relationship of TIM-1 and tumor-infiltrating lymphocytes (TILs) in cancer. Finally, we used immunohistochemistry to detect the expression of TIM-1 in tumor tissues.

\section{METHODS}

\section{Data Source and Processing}

Using the TIMER (Tumor Immune Estimation Resource, https:// cistrome.shinyapps.io/timer/) site to analyze the expression of TIM-1 in cancers (11), the mRNA profiling information is from the TCGA (The Cancer Genome Atlas, https://cancergenome. nih.gov/) database. We also use the Oncomine database to analyze the expression of TIM-1 in cancers (12). Bayes test was used to select TIM- 1 with a change $>=2$-fold and a $P$-value cutoff of 0.001 was defined as statistically significant.

\section{Survival Analysis}

GEPIA (Gene Expression Profiling Interactive Analysis, http:// gepia.cancer-pku.cn/) site was used to analyze the prognosis of TIM-1 in cancers by using the TCGA dataset (13). PrognoScan database (http://kmplot.com/analysis/) site was used to validate the prognosis of TIM-1 in cancers by using the GEO dataset (https://www.ncbi.nlm.nih.gov/) (14). A univariate Cox $P<0.05$ was defined as statistically significant. Moreover, we used the Kaplan-Meier plotter database to validate the prognosis of TIM-1 in cancers $(15,16)$.

\section{Immune Infiltration}

To reveal the immune infiltration of TIM-1 in cancer, we used the TISIDB (tumor-immune system interactions and drugbank, http://cis.hku.hk/TISIDB/index.php) database to infer the relations between abundance of tumor-infiltrating lymphocytes (TILs) and expression of TIM-1. The immunerelated signatures of 28 TIL types from Charoentong's study, which can be viewed in the download page. The relative abundance of TILs was inferred by using genomic variation analysis based on gene expression profiles (17).

\section{Immunofluorescence and Immunohistochemistry}

The TIM-1 distribution in cells and expression in cancer were reviewed by using the Human Protein Atlas (HPA, https://www. proteinatlas.org/) $(18,19)$. The TIM-1 distribution in cells was examined by immunofluorescence, and the protein expression was examined by immunohistochemistry.

\section{Statistical Analysis}

The distribution of TIM-1 in cancer was using HPA site, the expression of TIM-1 in cancer was using the TIMER and Oncomine databases. The survival curve was generated by GEPIA, PrognoScan and KaplanMeier diagrams. The results of KaplanMeier plots, PrognoScan, and GEPIA are displayed with $\mathrm{HR}$ and univariate Cox $P$-values from a log-rank test.

\section{RESULTS}

\section{TIM-1 Expression Profiles in Human Cancer Tissues}

To examine TIM-1 protein expression in human tumor tissues, the HPA database was used to assess the TIM-1 protein expression in human tumor tissues. As shown in Figure 1A, the TIM-1 mRNA expression was mainly in kidney, testis, and colon in normal human tissues. Then, we detected the TIM-1 protein expression in human tumor tissues by using the GTEx (Genotype-Tissue Expression) database, and TIM-1 protein expression was mainly in colorectal cancer, breast cancer, carcinoid, thyroid cancer, and prostate cancer (Figure 1B). Specifically, immunohistochemistry showed that TIM-1 protein expression was low in glandular cells in normal stomach tissues and normal lung tissues. In comparison, the TIM-1 was higher in expression in stomach cancer tissues and lung adenocarcinoma tissues, and distributed in both cytoplasma and cell membrane (Figures 1C-F). Next, we examined the association between TIM-1 expression and microsatellite instability (MSI). As shown in Figure 2F, higher TIM-1 expression was found in MSI tumors than genomically stable tumors in READ, KIRC, and UCEC in the TCGA dataset $(P<0.005)$. To inspect whether TIM-1 expression was related to the subtype of STAD, we divide STAD into five subtypes (CIN, EBV, HMSNV, HM-indel). We found high TIM-1 expression had no significant relation to the subtype of STAD $(P=0.667)$ (Figure 2G).

\section{The Landscape of TIM-1 Expression in Human Cancers}

Next, for analysis the different expressions of TIM-1 in tumor and normal tissues, the Oncomine database was used to analyze the TIM-1 mRNA levels in different cancers and normal tissues. As shown in Figure 2A, the TIM-1 expression was higher in breast cancer, kidney cancer, and ovarian cancer compared with the normal tissues. However, the TIM-1 expression was lower in colorectal cancer compared with the normal tissues. To further analyze TIM-1 in tumor and normal tissues, we compared the expression level of TIM-1 in the TCGA dataset. As shown in 


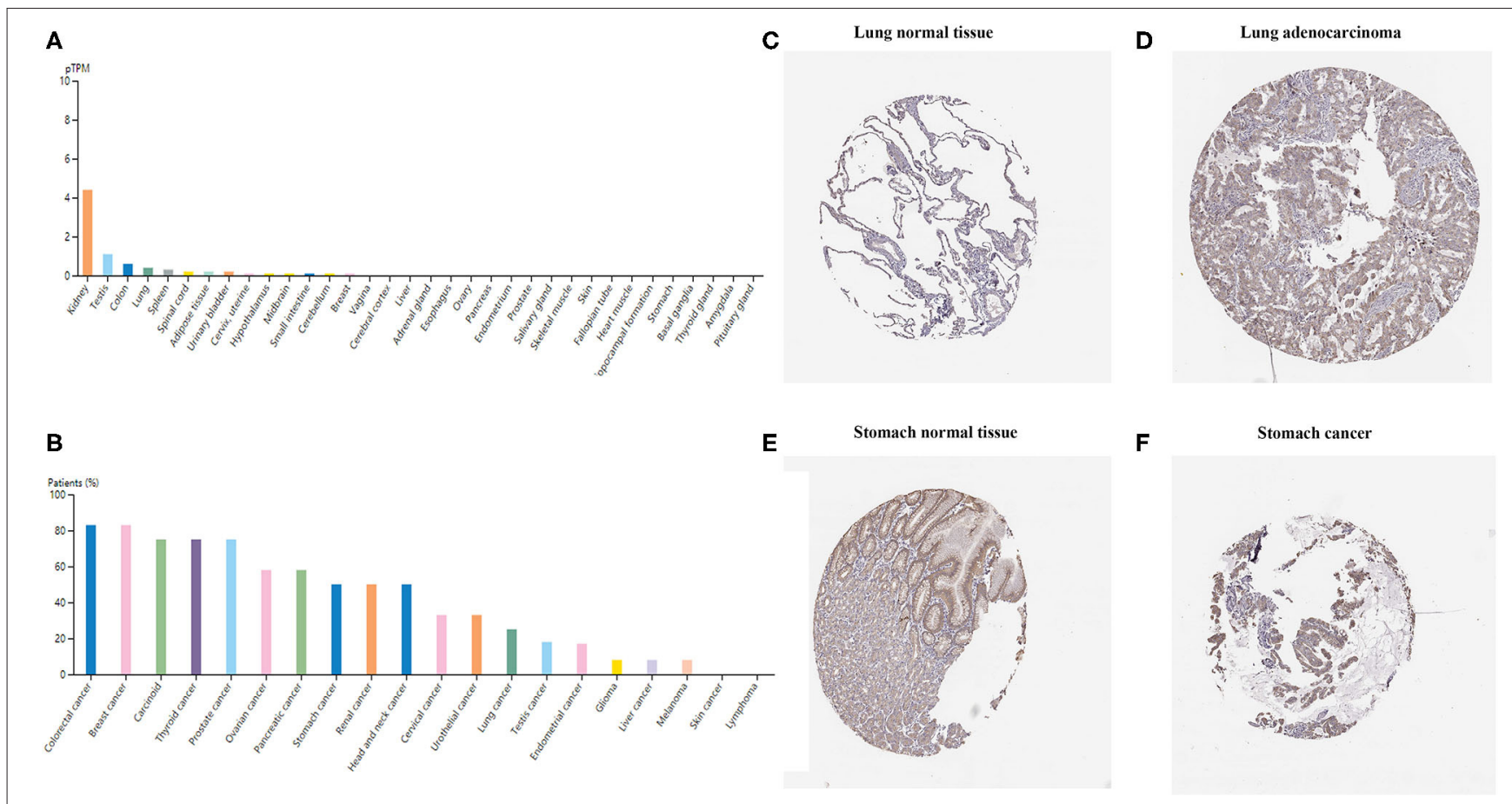

FIGURE 1 | TIM-1 expression profiles in human cancer tissues. (A) TIM-1 expression profiles in normal human tissues. (B) The protein expression profiles of TIM-1 in human cancer tissues. (C-F) Representative IHC images of TIM-1 expression in normal stomach tissues, stomach cancer tissues, normal lung tissues, and lung adenocarcinoma tissues.

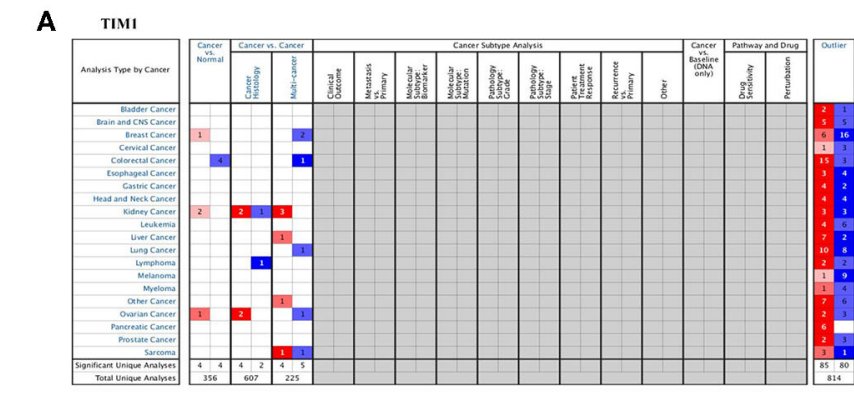

B

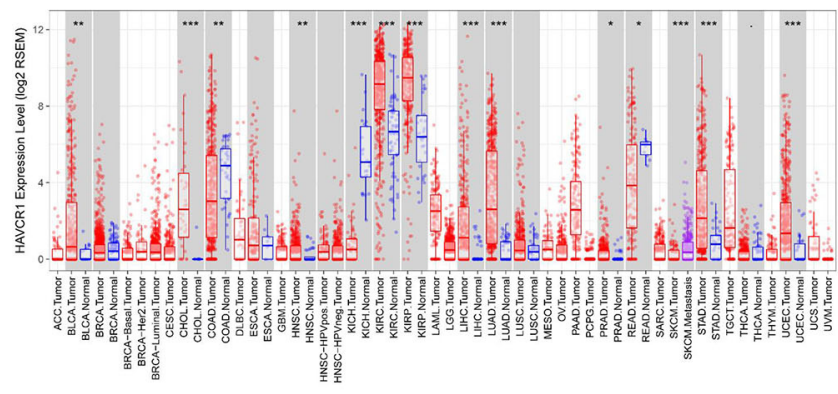

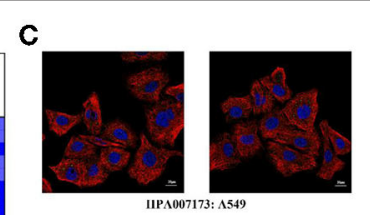

D
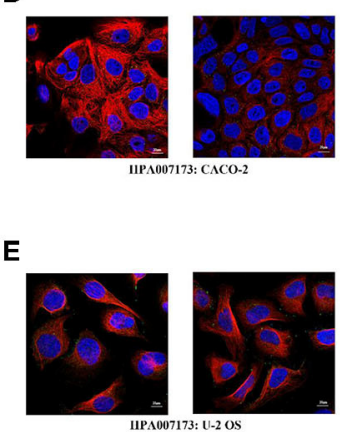
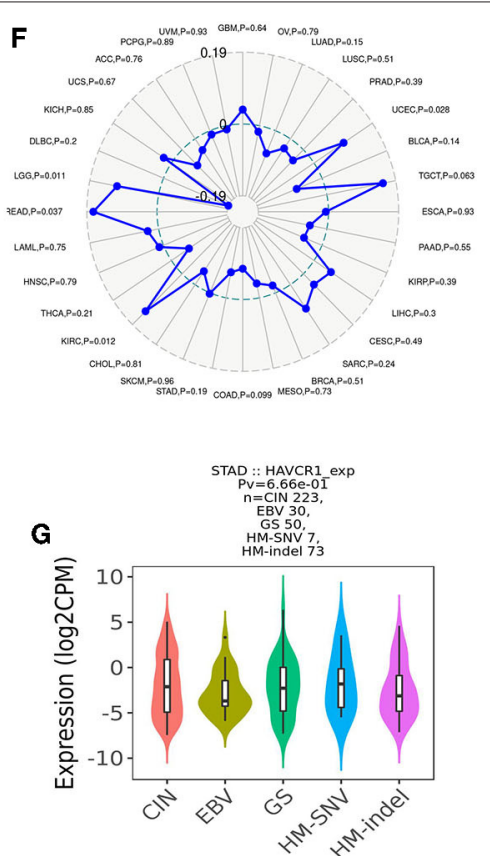

Subtype

FIGURE 2 | TIM-1 expression levels in different types of human cancers. (A) Increased or decreased TIM-1 in datasets of different cancers compared with normal tissues in the Oncomine database. (B) TIM-1 expression levels in different tumor types from TCGA database were determined by TIMER $\left({ }^{\star} P<0.05,{ }^{* \star} P<0.01,{ }^{\star \star *} P<\right.$ 0.001). (C-E) The distribution of TIM-1 in A549 cells, CACO-2 cells, and U-2 OS cells, blue represents nucleus, red represents microtubules, green represents antibody. (F) Correlation of TIM-1 expression and MSI in cancers. (G) Correlation of TIM-1 expression and molecular subtypes (CIN, EBV, HM-SNV, HM-indel) in STAD. 
Figure 2B, compared with normal tissues, the TIM-1 expression was significantly higher in bladder urothelial carcinoma (BLCA), cholangio carcinoma (CHOL), colon adenocarcinoma (COAD), head and Neck squamous cell carcinoma (HNSC), kidney renal clear cell carcinoma (KIRC), kidney renal papillary cell carcinoma (KIRP), liver hepatocellular carcinoma (LIHC), lung adenocarcinoma (LUAD), Prostate adenocarcinoma (PRAD), skin cutaneous melanoma (SKCM), stomach adenocarcinoma
(STAD), uterine corpus endometrial carcinoma(UCEC), and rectum adenocarcinoma (READ). However, TIM-1 expression was significantly lower in kidney chromophobe (KICH). In order to investigate the cellular localization of TIM-1 in cancer cells, we used the HPA database to examine the distribution of TIM-1 in cancer cells. As shown in Figures 2C-E, TIM-1 was mainly distributed in vesicles in A549 cells, CACO-2 cells, and U-2 OS cells.
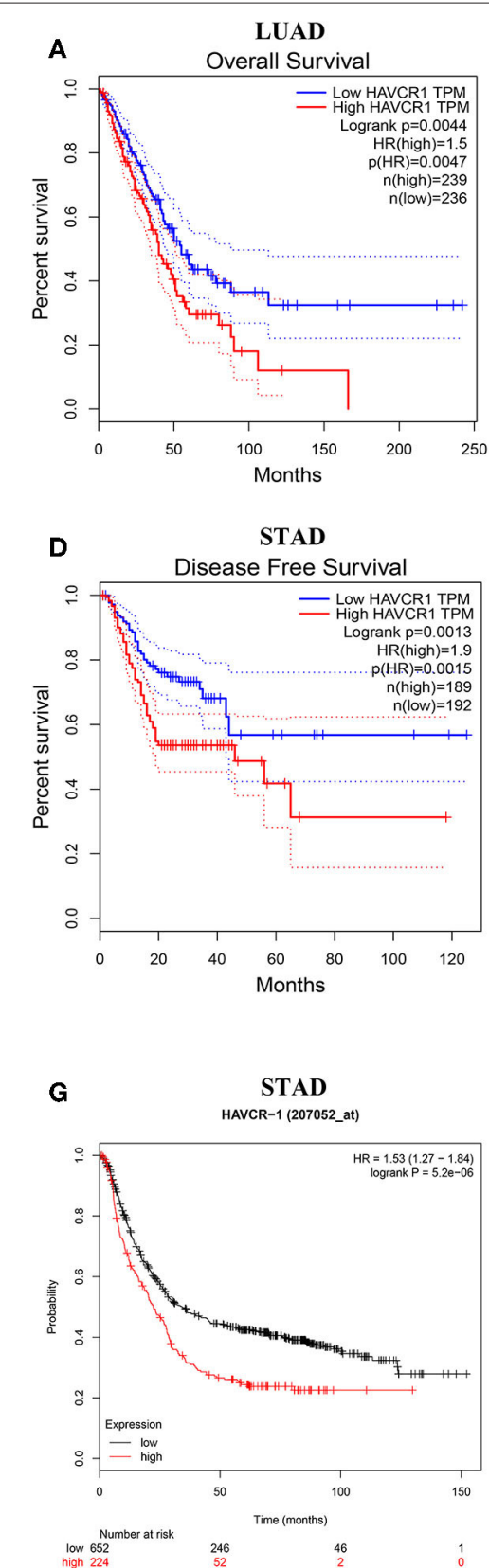

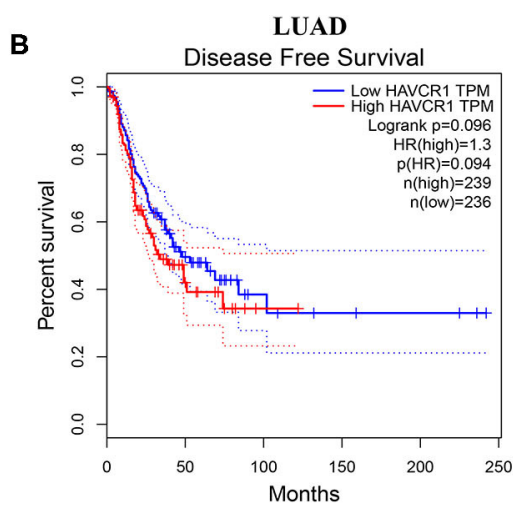

c

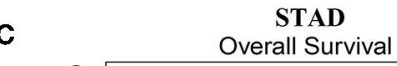

E

LUAD

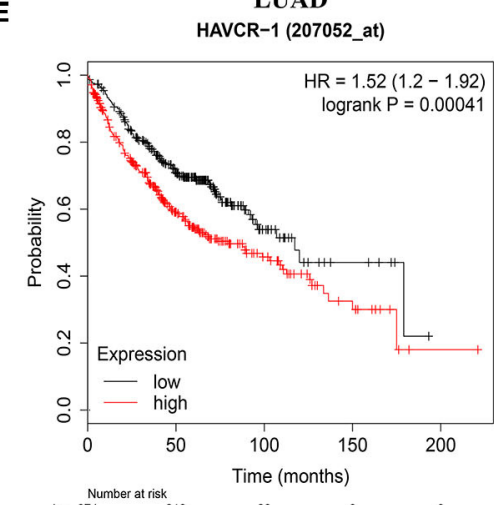

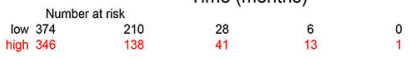

H

STAD

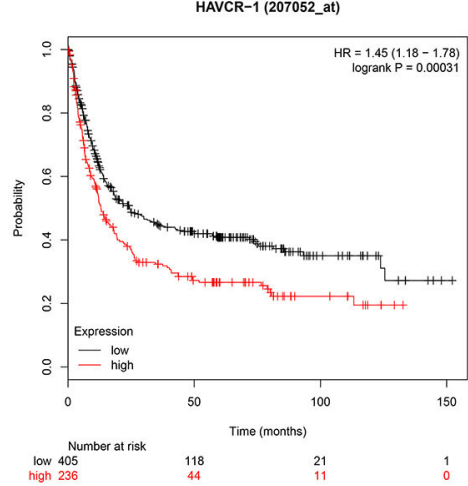

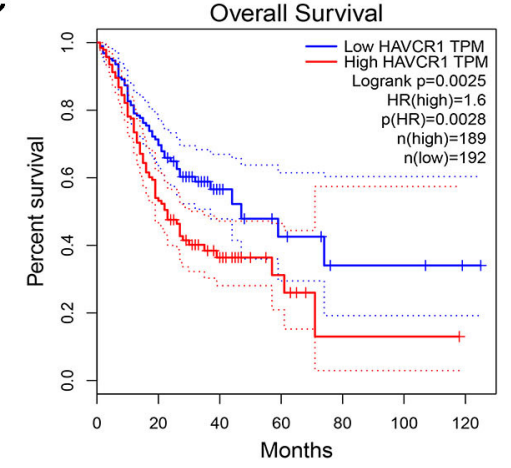

LUAD

$\mathbf{F}$

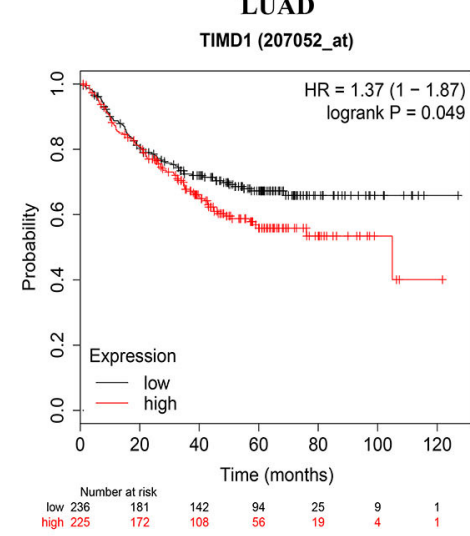

FIGURE 3 | Kaplan-Meier survival curves comparing the high and low expression of TIM-1 in different types of cancer in the TCGA dataset and GEO dataset. (A-D) Survival curves of OS and DFS in gastric cancer and lung adenocarcinoma in TCGA cohorts. (E,F) Survival curves of OS and PFS in six lung adenocarcinoma cohorts (GSE29013, GSE31210, GSE31908, GSE43580, GSE50081, GSE8894). (G,H) Survival curves of OS and PFS in six gastric cancer cohorts (GSE62254, GSE14210, GSE15459, GSE22377, GSE29272, GSE51105). 


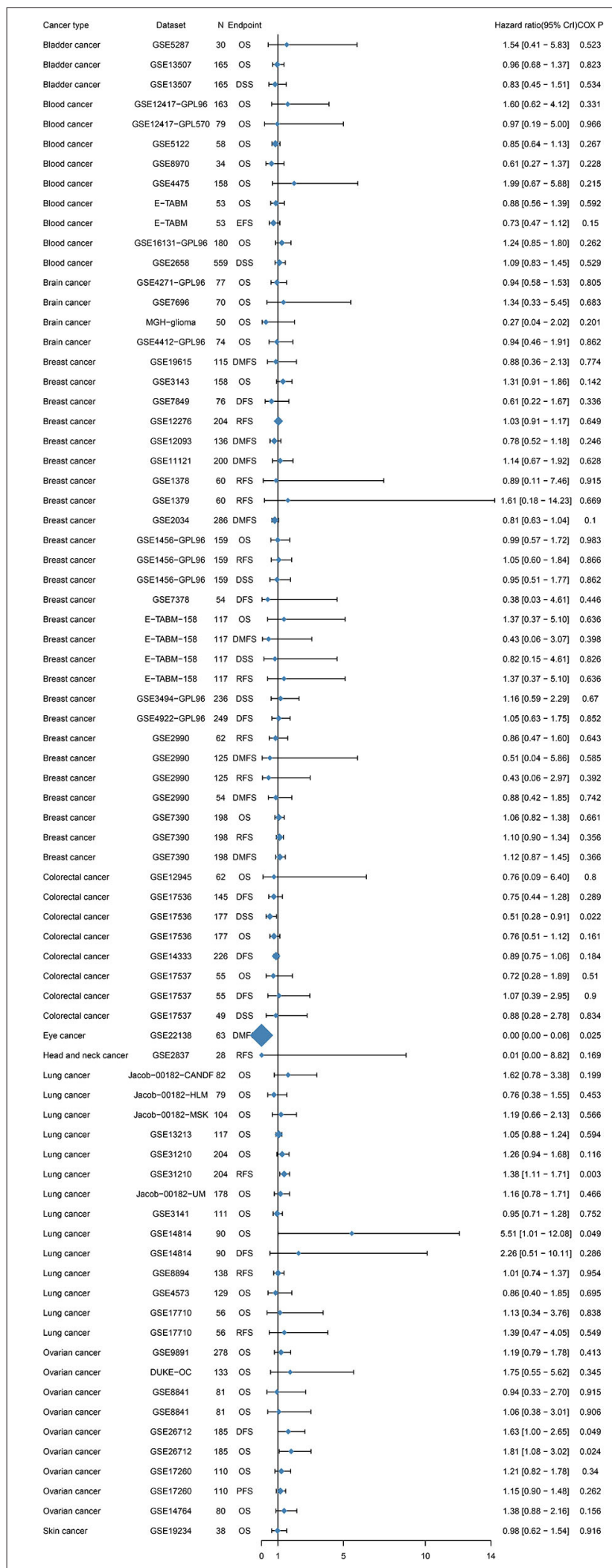

FIGURE 4 | Relation between TIM-1 expression and patient prognosis of different cancers in Prognoscan database.

\section{Survival Analysis of TIM-1 in Cancers}

Next, to inspect whether TIM-1 was related with prognosis in cancer patients, GEPIA site was used to analyze the prognosis of genes in cancers by using the TCGA dataset. Notably, high TIM1 expression levels were closely related with poorer prognosis of overall survival (OS) and disease-free survival (DFS) in stomach adenocarcinoma, OS in lung adenocarcinoma (Figures 3A-D). Meanwhile, high TIM-1 expression was closely related with better prognosis of DFS in BLCA, KIRC, OS in HNSC, KIRC, SKCM (Supplementary Figure 1).

To further examine the prognostic potential of TIM-1 in cancers, we used the PrognoScan database to examine the TIM1 in cancers. Three cohorts (GSE14814, GSE31210, GSE26712) included 90 samples, 204 samples and 185 samples in lung adenocarcinoma (20-22) and ovarian cancer, and showed that high TIM-1 expression was closely related with poorer prognosis (lung adenocarcinoma, OS HR $=5.51,95 \% \mathrm{CI}=1.01-12.08$, Cox $P=0.049$; DFS HR $=1.38,95 \% \mathrm{CI}=1.11-1.71$, Cox $P=0.003$; ovarian cancer, OS $\mathrm{HR}=1.81,95 \% \mathrm{CI}=1.08-$ 3.02, Cox $P=0.024$; DFS HR $=1.63$, 95\% CI $=1.00-2.65$, Cox $P=0.049$ ). Moreover, two cohorts (GSE17536, GSE22138) included 177 samples and 63 samples in colorectal cancer (23) and eye cancer (24), and indicated that high TIM-1 expression was closely related with better prognosis (colorectal cancer, DSS $\mathrm{HR}=0.51,95 \% \mathrm{CI}=0.28-0.91$, Cox $P=0.022$; eye cancer, DMFS HR $=0.00,95 \%$ CI $=0.00-0.06$, Cox $P=0.025)$ (Figure 4).

To validate the prognostic potential of TIM-1 in stomach adenocarcinoma and lung adenocarcinoma, we used the KaplanMeier plotter database to validate the prognostic potential of TIM-1 in stomach adenocarcinoma and lung adenocarcinoma. Interestingly, six cohorts [GSE14210 (25), GSE15459 (26), GSE22377 (27), GSE29272 (28), GSE51105 (29), GSE62254 (30)] included 882 samples in stomach adenocarcinoma and indicated that high TIM-1 expression was closely related with poorer prognosis (OS $\mathrm{HR}=1.53,95 \% \mathrm{CI}=1.27-1.84$, $P<0.001 ;$ PFS HR $=1.45,95 \%$ CI $=1.18-1.78, P<$ 0.001) (Figures 3G,H). Moreover, six cohorts [GSE29013 (31), GSE31210 (32), GSE31908 (33), GSE43580 (34), GSE50081 (35), GSE8894 (36)], which included 866 samples in lung adenocarcinoma and showed that high TIM-1 expression was closely related with poorer prognosis (OS HR $=1.52,95 \%$ $\mathrm{CI}=1.20-1.92, P<0.001$; PFS HR $=1.37,95 \% \mathrm{CI}=1.00-1.87$, $P=0.049$ ) (Figures 3E,F).

\section{High Expression of TIM-1 Correlates With Clinical Relevance of Stomach Adenocarcinoma and Lung Adenocarcinoma}

Next, we examined the association between the TIM-1 expression and the clinical relevance of stomach cancer and lung adenocarcinoma patients. As shown in Table 1, high TIM-1 expression was closely related with poorer prognosis in females (OS HR $=1.93, P<0.001$; PFS HR $=1.54, P=0.035)$ and males (OS HR $=1.54, P<0.001$; PFS HR $=1.53, P<0.001$ ). 
TABLE 1 | Correlation of TIM1 mRNA expression and clinical prognosis in gastric cancer with different clinicopathological factors by Kaplan-Meier plotter (GSE62254, GSE14210, GSE15459, GSE22377, GSE29272, GSE51105).

\begin{tabular}{|c|c|c|c|c|c|c|}
\hline \multirow[t]{2}{*}{ Clinicopathological characteristics } & \multicolumn{3}{|c|}{ Overall survival $(n=882)$} & \multicolumn{3}{|c|}{ Progression-free survival $(n=646)$} \\
\hline & $N$ & Hazard ratio & $\boldsymbol{P}$ & $N$ & Hazard ratio & $\boldsymbol{P}$ \\
\hline \multicolumn{7}{|l|}{ Sex } \\
\hline Female & 244 & $1.93(1.34-2.79)$ & $3.4 \mathrm{e}-05$ & 244 & $1.54(1.03-2.30)$ & 0.035 \\
\hline Male & 567 & $1.54(1.23-1.94)$ & $1.9 e-05$ & 567 & $1.53(1.21-1.95)$ & 4.1e-05 \\
\hline \multicolumn{7}{|l|}{ Stage } \\
\hline 1 & 69 & $3.36(0.95-11.88)$ & 0.047 & 69 & $2.88(0.78-10.63)$ & 0.097 \\
\hline 2 & 145 & $1.86(1.02-3.39)$ & 0.041 & 145 & $1.88(1.02-3.46)$ & 0.041 \\
\hline 3 & 319 & $2.19(1.52-3.13)$ & $1.3 e-05$ & 319 & $1.97(1.32-2.94)$ & $6.7 e-05$ \\
\hline 4 & 152 & $1.15(0.76-1.74)$ & 0.500 & 152 & $0.78(0.51-1.21)$ & 0.270 \\
\hline \multicolumn{7}{|l|}{ Stage T } \\
\hline 2 & 253 & 1.53 (1.00-2.36) & 0.049 & 239 & $1.61(1.06-2.43)$ & 0.023 \\
\hline 3 & 208 & $1.58(1.10-2.27)$ & 0.013 & 204 & 1.57 (1.11-2.23) & 0.011 \\
\hline 4 & 39 & $0.25(0.10-0.65)$ & 0.002 & 39 & $0.26(0.10-0.69)$ & 0.003 \\
\hline \multicolumn{7}{|l|}{ Stage N } \\
\hline 0 & 76 & $3.26(0.97-10.99)$ & 0.044 & 72 & $3.22(0.96-10.86)$ & 0.046 \\
\hline 1 & 232 & 2.03 (1.34-3.07) & $6 e-04$ & 222 & $2.09(1.41-3.09)$ & $1.7 e-05$ \\
\hline 2 & 129 & $1.73(1.10-2.72)$ & 0.016 & 125 & $1.65(1.07-2.54)$ & 0.022 \\
\hline 3 & 76 & $1.38(0.78-2.46)$ & 0.270 & 76 & 0.77 (0.46-1.30) & 0.330 \\
\hline $1+2+3$ & 437 & 1.48 (1.14-1.93) & $3.6 e-04$ & 423 & $1.48(1.14-1.91)$ & 0.003 \\
\hline \multicolumn{7}{|l|}{ Stage M } \\
\hline 0 & 459 & $1.62(1.22-2.15)$ & $6.8 e-05$ & 443 & $1.64(1.25-2.15)$ & $3.1 e-05$ \\
\hline 1 & 58 & $2.49(1.26-4.92)$ & $6.6 e-04$ & 56 & $2.49(1.19-5.21)$ & 0.013 \\
\hline \multicolumn{7}{|l|}{ Lauren classification } \\
\hline Intestinal & 336 & 2.29 (1.65-3.19) & $3.9 e-07$ & 263 & 1.87 (1.30-2.68) & $6.2 \mathrm{e}-05$ \\
\hline Diffuse & 248 & $1.48(1.05-2.07)$ & 0.024 & 231 & 1.48 (1.03-2.13) & 0.035 \\
\hline \multicolumn{7}{|l|}{ Differentiation } \\
\hline Poor & 166 & $1.41(0.94-2.11)$ & 0.092 & 121 & $0.76(0.48-1.20)$ & 0.235 \\
\hline Moderate & 67 & $1.80(0.86-3.75)$ & 0.110 & 67 & $1.84(0.91-3.71)$ & 0.083 \\
\hline \multicolumn{7}{|l|}{ Perforation } \\
\hline No & 169 & $1.15(0.77-1.73)$ & 0.490 & 169 & $1.19(0.75-1.87)$ & 0.459 \\
\hline Yes & 4 & - & - & 4 & - & - \\
\hline \multicolumn{7}{|l|}{ Treatment } \\
\hline Surgery alone & 393 & $1.41(1.06-1.88)$ & 0.019 & 375 & 1.40 (1.06-1.85) & 0.019 \\
\hline 5 FU based adjuvant & 158 & 1.35 (0.92-1.98) & 0.130 & 153 & $1.46(0.98-2.19)$ & 0.063 \\
\hline Other adjuvant & 80 & $1.97(0.81-4.84)$ & 0.130 & 80 & 1.95 (0.89-4.29) & 0.088 \\
\hline \multicolumn{7}{|l|}{ HER2 status } \\
\hline Negative & 641 & $1.36(1.09-1.70)$ & 0.007 & 408 & $1.36(1.04-1.76)$ & 0.021 \\
\hline Positive & 425 & 1.52 (1.12-2.05) & 0.006 & 233 & 1.62 (1.15-2.29) & 0.006 \\
\hline
\end{tabular}

Moreover, high TIM-1 expression was closely related with poorer OS and PFS in stage 2 (OS HR $=1.86, P=0.041$; PFS HR $=1.88$, $P=0.041)$ and 3 (OS HR $=2.19, P<0.001$; PFS HR $=1.97$, $P<0.001)$ of stomach cancer patients, and poorer OS in stage 1 (HR $=3.36, P=0.047)$, but was not related with OS and PFS in stage 4 (OS HR $=1.15, P=0.500$; PFS HR $=0.78, P=0.270)$, and PFS in stage $1(\mathrm{HR}=2.88, P=0.097)$. Furthermore, high TIM1 expression was marginally associated with poorer prognosis in the $4 \mathrm{~N}$ categories. In addition, high TIM-1 expression was closely related to poorer prognosis in the lauren classification, moderate differentiation, negative and positive HER-2 status. As shown in Table 2, high TIM-1 expression was closely related to poorer OS in males $(\mathrm{HR}=1.53, P=0.011)$, but was not associated with $\mathrm{OS}$ and PFS in females (OS HR $=1.33, P=0.144$; PFS HR $=1.33$, $P=0.221)$, and PFS males ( $\mathrm{HR}=1.46, P=0.088)$. Moreover, high TIM-1 expression was not correlated with poorer prognosis in other clinical characteristics (smoking history, stage, and lymph node metastasis), which may be due to lack of sufficient data to analyze.

\section{TIM-1 Expression Was Correlated With TILs}

TILs are an independent predictor in cancers $(37,38)$. Therefore, the TISIDB database was used to infer the relations between abundance of TILs and expression of TIM-1. The landscape of 
TABLE 2 | Correlation of TIM1 mRNA expression and clinical prognosis in lung adenocarcinoma with different clinicopathological factors by Kaplan-Meier plotter (GSE29013, GSE31210, GSE31908, GSE43580, GSE50081, GSE8894).

\begin{tabular}{|c|c|c|c|c|c|c|}
\hline \multirow[t]{2}{*}{ Clinicopathological characteristics } & \multicolumn{3}{|c|}{ Overall survival $(n=866)$} & \multicolumn{3}{|c|}{ Progression-free survival $(n=461)$} \\
\hline & $N$ & Hazard ratio & $\boldsymbol{P}$ & $N$ & Hazard ratio & $P$ \\
\hline \multicolumn{7}{|l|}{ Sex } \\
\hline Female & 318 & $1.33(0.91-1.96)$ & 0.144 & 235 & $1.33(0.84-2.09)$ & 0.221 \\
\hline Male & 344 & $1.53(1.10-2.13)$ & 0.011 & 226 & $1.46(0.94-2.25)$ & 0.088 \\
\hline \multicolumn{7}{|l|}{ Smoking history } \\
\hline No & 143 & $1.41(0.63-3.17)$ & 0.400 & 143 & $1.67(0.90-3.09)$ & 0.101 \\
\hline Yes & 246 & $1.22(0.77-1.95)$ & 0.397 & 243 & $1.38(0.89-2.14)$ & 0.145 \\
\hline \multicolumn{7}{|l|}{ Stage } \\
\hline 1 & 370 & $1.84(1.24-2.72)$ & 0.002 & 283 & $1.24(0.77-2.00)$ & 0.374 \\
\hline 2 & 136 & $1.69(1.03-2.77)$ & 0.035 & 103 & $1.41(0.81-2.46)$ & 0.221 \\
\hline 3 & 24 & $0.92(0.33-2.56)$ & 0.872 & 10 & - & - \\
\hline 4 & 4 & - & - & 0 & - & - \\
\hline \multicolumn{7}{|l|}{ Stage $\mathbf{T}$} \\
\hline 1 & 123 & $1.00(0.52-1.82)$ & 0.999 & 47 & $7.15(0.86-59.53)$ & 0.034 \\
\hline 2 & 105 & $1.04(0.60-1.79)$ & 0.898 & 93 & $0.98(0.52-1.83)$ & 0.942 \\
\hline 3 & 4 & - & - & 2 & - & - \\
\hline \multicolumn{7}{|l|}{ Stage $\mathbf{N}$} \\
\hline 0 & 184 & $0.80(0.49-1.30)$ & 0.366 & 102 & $0.53(0.24-1.20)$ & 0.121 \\
\hline 1 & 44 & $1.64(0.74-3.63)$ & 0.216 & 38 & $3.41(1.22-9.57)$ & 0.013 \\
\hline 2 & 3 & - & - & 25 & - & - \\
\hline \multicolumn{7}{|l|}{ Stage M } \\
\hline 0 & 231 & $0.95(0.64-1.41)$ & 0.799 & 142 & $1.16(0.65-2.05)$ & 0.616 \\
\hline 1 & 1 & - & - & 0 & - & - \\
\hline \multicolumn{7}{|l|}{ Chemotherapy } \\
\hline No & 21 & $1.32(0.32-5.37)$ & 0.699 & 11 & - & - \\
\hline Yes & 36 & $2.84(0.74-10.86)$ & 0.112 & 19 & - & - \\
\hline
\end{tabular}

the relationship between TIM-1 expression and TILs in different types of cancer was shown in Figure 5A. The relations between abundance of 28 TIL types and expression of TIM-1 was weakly to moderately correlated. Specifically, TIM-1 expression was positively closely related with infiltrating levels of CD56dim natural killer cell in lung adenocarcinoma $(r=0.107, P=0.015)$ and monocyte in stomach cancer $(r=0.122, P=0.013)$, and was negatively correlated with infiltrating levels of natural killer cell $(r=-0.090, P=0.040)$, gamma delta $\mathrm{T}$ cell $(r=-0.090, P=0.042)$, and regulatory $\mathrm{T}$ cell $(r=-0.090$, $P=0.041$ ) in lung adenocarcinoma (Figures 5B-F). Next, we detected the associations between TIM-1 expression and immune subtypes across human cancers, and the landscape of relationship between TIM-1 expression and immune subtypes across human cancers was shown in Figure 5G. Specifically, TIM-1 expression was not correlated with immune subtypes (wound healing, IFN-gamma dominant, inflammatory, lymphocyte depleted, TGF- $\beta$ dominant) in stomach cancer and lung adenocarcinoma (Figures 5H,I).

\section{DISCUSSION}

Due to advances in treatment, the mortality rate of tumors has been declining in recent years, a large part of which is due to immunotherapy (39). Immunotherapy represented by anti-PD-1/PD-L1 monoclonal antibody drugs and CAR-T cell therapy has attracted much attention, and encouraging results have continued. Both of them are essentially the ability of the human autoimmune system to recruit and activate human core immune guardian-T cells to identify and clear cancer cells through antigen-antibody response (1). However, not every patient responds to this treatment, especially in gastric cancer $(40,41)$. Therefore, there is an urgent need to clarify and identify new immune-related therapeutic targets. High throughput technology has been widely employed to investigate gene expression in numerous tumors, providing a novel method to identify significant genes and explore tumor progression and initiation.

Here, we report that high TIM-1 expression was observed in bladder, cholangio, head and neck, colorectal, gastric, kidney, liver, lung adenocarcinoma, skin, uterine corpus endometrial, and pancreatic cancers compared to the normal tissues, and immunofluorescence shows that TIM-1 is mainly localized in vesicles. Simultaneously, high TIM-1 expression was closely related with poorer overall survival in gastric, lung adenocarcinoma, and poorer disease-specific survival in gastric cancer in TCGA cohort. High TIM-1 expression was closely related with poorer overall survival in gastric and lung 


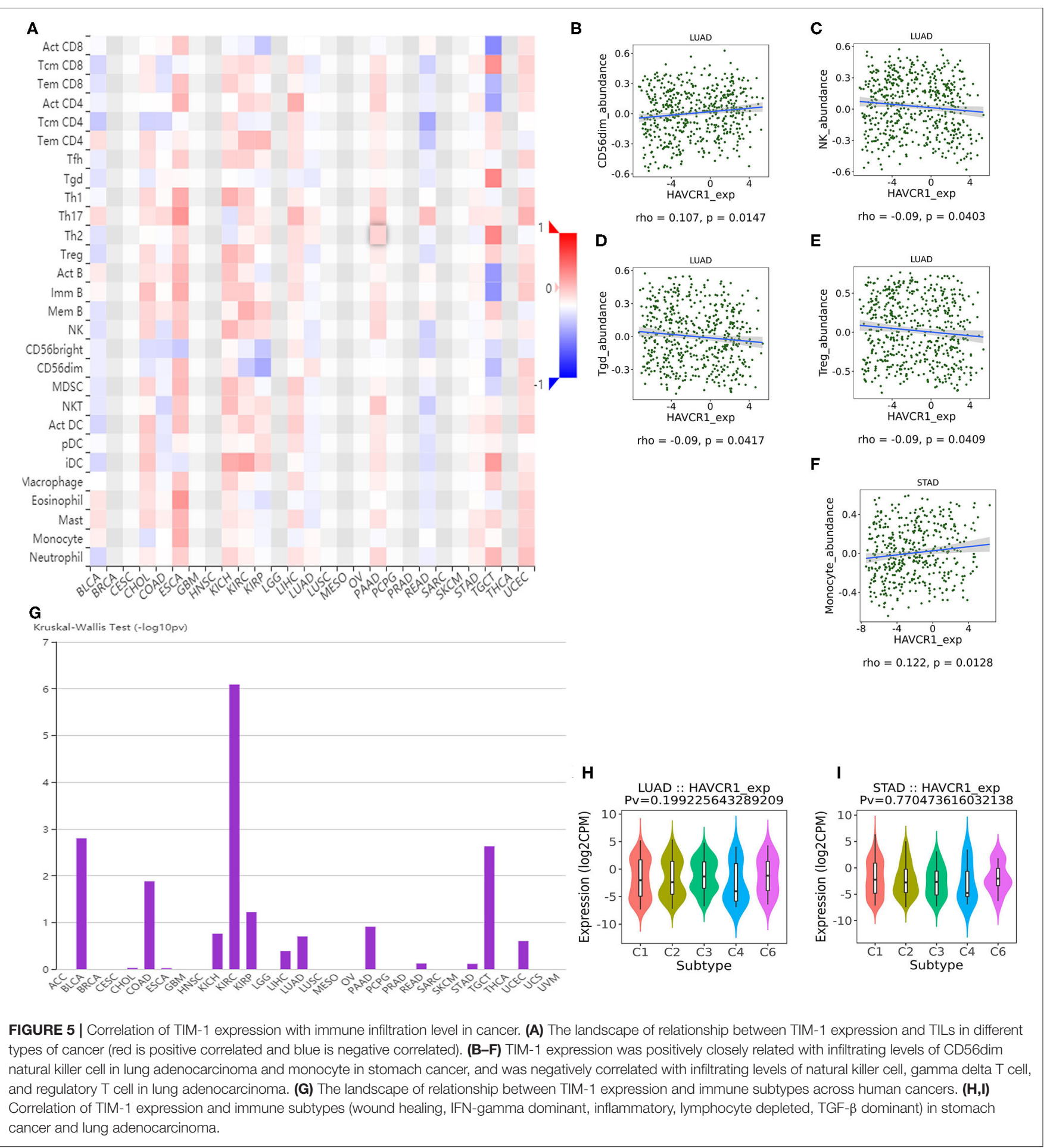

adenocarcinoma, and poorer disease-specific survival in gastric cancer, lung adenocarcinoma was validated in GEO database. Moreover, high expression of TIM-1 correlates with clinical relevance of gastric cancer and lung adenocarcinoma.

TIM-1 has a certain value in evaluating the disease progression and survival prognosis of patients with cancer (42). TIM-1 expression level in non-small-cell lung cancer is significantly correlated with tumor size, degree of differentiation, clinical stage, lymph node, and distant metastasis. Moreover, the overall survival rate of non-small-cell lung cancer patients with high expression of TIM-1 is significantly lower than that of patients with low expression of TIM-1. In cell experiments, it was also 
found that inhibition of TIM-1 expression could inhibit the migration and invasion ability of A549 and SK-MES- 1 cells in vitro, respectively (43). All these suggest that TIM-1 plays an important role in the invasion and metastasis of cancers.

We also investigated the relationship between immune infiltration and TIM in cancer, and found TIM-1 was positively associated with tumor-infiltrating lymphocytes of CD56dim natural killer cell in lung adenocarcinoma and monocyte in gastric cancer, and was negatively correlated with infiltrating levels of natural killer cell, gamma delta $\mathrm{T}$ cell, and regulatory $\mathrm{T}$ cell in lung adenocarcinoma. Finally, immunohistochemistry shows TIM-1 expression was higher in lung adenocarcinoma and gastric cancer compared to the normal tissues. Thus, our study provides us clues to understand the potential role of TIM1 in tumor immunology and may be a potential prognostic molecular marker. TIM-1 mainly provides stimulating signals for the activation of $\mathrm{T}$ cells, participates in the proliferation and differentiation of $\mathrm{T}$ cells, and inhibits the occurrence of peripheral tolerance (44). When Th cells differentiate into Th1 and Th2 cells, TIM-1 is only highly expressed on Th2 cells and has been shown to have an important relationship with mouse Th2 cell-mediated airway hyperreactive diseases (45). There is evidence that when $\mathrm{T}$ cells are stimulated, the tyrosine residue of TIM-1 protein extending into the cell is phosphorylated, which is also the promoter of IL-4, and an activating nuclear factor of $\mathrm{T}$ cells/activator protein-1 (NFAT/AP-1) dependent transcription provides costimulatory signals (46). The regulation of cytokine transcription is controlled by many transcription factors, among which NFAT is one of the most thoroughly studied transcription factors (47). It has been proved that four members of NFAT are expressed in lymphocytes (48). The activity of most members of the NFAT family is regulated by $\mathrm{Ca}^{2+}$. When the concentration of $\mathrm{Ca}^{2+}$ in the cell increases, dephosphorization mediated by $\mathrm{Ca}^{2+} /$ calmodulin phosphatase occurs, and NFAT molecules can enter the nucleus to play a role. Furthermore, the activation of NFAT molecule follows the activation of $\mathrm{Ca}^{2+} /$ calmodulin dependent phosphatase, while in $\mathrm{T}$ cells, the level of free $\mathrm{Ca}^{2+}$ is higher, so the activity of $\mathrm{Ca}^{2+} /$ calmodulin dependent phosphatase increases, and NFAT can maintain the activation state in the nucleus for a long time and promote the transcription of some genes. In addition to $\mathrm{Ca}^{2+} /$ calmodulin dependent phosphatase, there are many proteins and signaling pathways that regulate NFAT, such as $\mathrm{T}$ cell receptor (TCR) cross-linking (49-51). Moreover, the activation of Lck and ZAP70 leads to calcium mobilization in T cells, which leads to NFAT-dependent reporter gene expression (52). Experiments have shown that the cells co-expressing TIM1 and NFAT/AP-1 responded more strongly to the stimulation from TCR/CD3 complex, while the activity of NFAT/AP-1 did not increase, indicating that TIM-1 may play a costimulatory role in NFAT/AP-1-dependent transcription (53).

It has been reported that the use of a TIM- 1 specific antibody in a mouse asthma model can inhibit the occurrence and severity of airway hyperreactive inflammation by reducing the production of cytokines such as IL-10 and IL-13, and earlier studies have also mentioned that a positive hepatitis A virus reduces an individual's susceptibility to certain allergic diseases (54). Therefore, it can be inferred that the interaction between TIM-1 and ligand can enhance the activation of $\mathrm{T}$ cells and increase the production of Th2 type cytokines, while blocking this interaction can greatly inhibit the activity of Th2 cells, thus regulating the immune response mediated by Th2 cells. Therefore, TIM-1 plays an important role in regulating the differentiation, proliferation, and effector function of Th cells. The action of TIM- 1 coding products and ligands can promote the differentiation and proliferation of Th cells and enhance the immunity of Th2 cells (55). Through the study of the TIM-1 gene, we can have a deeper understanding of all kinds of inflammatory responses mediated by Th cells (such as asthma, allergic rhinitis, and other autoimmune diseases) and the mechanism of cancer, so as to have a far-reaching impact on the prevention and treatment of these diseases.

There are some limitations to this study. Firstly, there is no experimental validation of the predicted results, and the authors should pay attention to the experimental validation of the predicted results by different methods to be further confirmed, for example by RT-PCR in addition to highthroughput sequencing. TIM- 1 is down-regulated in $\mathrm{KICH}$ and a protective effect was detected in colorectal cancer and eye cancer as demonstrated in two cohorts (GSE17536, GSE22138). The included studies did not cover all previous published literatures revolved in TIM-1 and certain cancers with controversial findings. Therefore, experimental validation of the predicted results is still needed by RT-PCR to be further confirmed.

In summary, we applied integrated bioinformatics approaches to suggest that high TIM-1 was closely related with poor prognosis in gastric cancer and lung adenocarcinoma. Therefore, TIM-1 can be used as a prognostic biomarker in gastric cancer and lung adenocarcinoma, which might provide a novel direction to explore the pathogenesis of gastric and lung adenocarcinoma.

\section{DATA AVAILABILITY STATEMENT}

Publicly available datasets were analyzed in this study. This data can be found here: https://cancergenome.nih.gov/.

\section{AUTHOR CONTRIBUTIONS}

$\mathrm{XK}$ and $\mathrm{HJ}$ designed the research, analyzed the data, and wrote the paper. $\mathrm{MF}$ and $\mathrm{XN}$ performed the data analysis and interpreted the data. All authors read and approved the final manuscript.

\section{SUPPLEMENTARY MATERIAL}

The Supplementary Material for this article can be found online at: https://www.frontiersin.org/articles/10.3389/fonc. 2020.01086/full\#supplementary-material

Supplementary Figure 1 | Correlation of TIM-1 expression with prognostic values in diverse types of cancer. 


\section{REFERENCES}

1. Miller KD, Nogueira L, Mariotto AB, Rowland JH, Yabroff KR, Alfano CM, et al. Cancer treatment and survivorship statistics, (2019). CA: Cancer J Clin. (2019) 69:363-85. doi: 10.3322/caac.21565

2. Siegel RL, Miller KD, Jemal A. Cancer statistics, (2019). CA: Cancer J Clin. (2019) 69:7-34. doi: 10.3322/caac.21551

3. El-Deiry WS, Goldberg RM, Lenz HJ, Shields AF, Gibney GT, Tan AR, et al. The current state of molecular testing in the treatment of patients with solid tumors, (2019). CA: Cancer J Clin. (2019) 69:305-43. doi: 10.3322/caac.21560

4. immunology RCJS. A new perspective in cancer immunotherapy: PD-1 on myeloid cells takes center stage in orchestrating immune checkpoint blockade. Sci Immunol. (2020) 5:eaaz8128. doi: 10.1126/sciimmunol.aaz8128

5. Grosser R, Cherkassky L, Chintala N, Adusumilli PS. Combination immunotherapy with CAR $\mathrm{T}$ cells and checkpoint blockade for the treatment of solid tumors. Cancer Cell. (2019) 36:47182. doi: 10.1016/j.ccell.2019.09.006

6. Evans JP, Liu SL. Multifaceted roles of TIM-Family proteins in virus-host interactions. Trends Microbiol. (2019) 28:22435. doi: 10.1016/j.tim.2019.10.004

7. Chu LW, Yang CJ, Peng KJ, Chen PL, Wang SJ, Ping YH. TIM-1 as a signal receptor triggers dengue virus-induced autophagy. Int J Mol Sci. (2019) 20:4893. doi: 10.3390/ijms20194893

8. Guo H, Shen Y, Kong YH, Li S, Jiang R, Liu C, et al. The expression of Tim-1 and Tim-4 molecules in regulatory T cells in type 1 diabetes. Endocrine. (2020) 68:64-70. doi: 10.1007/s12020-019-02173-8

9. Zheng Y, Wang L, Chen M, Liu L, Pei A, Zhang R, et al. Inhibition of $\mathrm{T}$ cell immunoglobulin and mucin-1 (TIM-1) protects against cerebral ischemia-reperfusion injury. Cell Commun Signal. (2019). 17:103. doi: 10.1186/s12964-019-0417-4

10. Ye L, Zhang Q, Cheng Y, Chen X, Wang G, Shi M, et al. Tumor-derived exosomal HMGB1 fosters hepatocellular carcinoma immune evasion by promoting TIM-1 regulatory B cell expansion. J Immunother Cancer. (2018). 6:145. doi: 10.1186/s40425-018-0451-6

11. Li T, Fan J, Wang B, Traugh N, Chen Q, Liu JS, et al. TIMER: a web server for comprehensive analysis of tumor-infiltrating immune cells. Cancer Res. (2017) 77:e108-10. doi: 10.1158/0008-5472.CAN-17-0307

12. Rhodes DR, Kalyana-Sundaram S, Mahavisno V, Varambally R, Yu J, Briggs BB, et al. Oncomine 3.0: genes, pathways, and networks in a collection of 18,000 cancer gene expression profiles. Neoplasia. (2007) 9:16680. doi: $10.1593 /$ neo.07112

13. Tang Z, Li C, Kang B, Gao G, Li C, Zhang Z. GEPIA: a web server for cancer and normal gene expression profiling and interactive analyses. Nucleic Acids Res. (2017) 45:W98-102. doi: 10.1093/nar/gkx247

14. Mizuno H, Kitada K, Nakai K, Sarai A. PrognoScan: a new database for meta-analysis of the prognostic value of genes. BMC Med Genom. (2009) 2:18. doi: 10.1186/1755-8794-2-18

15. Szasz AM, Lanczky A, Nagy A, Forster S, Hark K, Green JE, et al. Cross-validation of survival associated biomarkers in gastric cancer using transcriptomic data of 1,065 patients. Oncotarget. (2016) 7:4932233. doi: 10.18632/oncotarget.10337

16. Gyorffy B, Surowiak P, Budczies J, Lanczky A. Online survival analysis software to assess the prognostic value of biomarkers using transcriptomic data in non-small-cell lung cancer. PLoS ONE. (2013) 8:e82241. doi: 10.1371/journal.pone.0082241

17. $\mathrm{Ru} \mathrm{B}$, Wong CN, Tong Y, Zhong JY, Zhong SSW, Wu WC, et al. TISIDB: an integrated repository portal for tumor-immune system interactions. Bioinformatics. (2019) 35:4200-2. doi: 10.1093/bioinformatics/btz210

18. Uhlen M, Fagerberg L, Hallstrom BM, Lindskog C, Oksvold P, Mardinoglu A, et al. Proteomics. tissue-based map of the human proteome. Science. (2015) 347:1260419. doi: 10.1126/science.1260419

19. Uhlen M, Oksvold P, Fagerberg L, Lundberg E, Jonasson K, Forsberg M, et al. Towards a knowledge-based human protein atlas. Nat Biotechnol. (2010) 28:1248-50. doi: 10.1038/nbt1210-1248

20. Zhu CQ, Ding K, Strumpf D, Weir BA, Meyerson M, Pennell N, et al. Prognostic and predictive gene signature for adjuvant chemotherapy in resected non-small-cell lung cancer. J Clin Oncol. (2010) 28:441724. doi: $10.1200 /$ JCO.2009.26.4325
21. Yamauchi M, Yamaguchi R, Nakata A, Kohno T, Nagasaki M, Shimamura $\mathrm{T}$, et al. Epidermal growth factor receptor tyrosine kinase defines critical prognostic genes of stage I lung adenocarcinoma. PLoS ONE. (2012) 7:e43923. doi: 10.1371/journal.pone.0043923

22. Bonome T, Levine DA, Shih J, Randonovich M, Pise-Masison CA, Bogomolniy F, et al. A gene signature predicting for survival in suboptimally debulked patients with ovarian cancer. Cancer Res. (2008) 68:547886. doi: 10.1158/0008-5472.CAN-07-6595

23. Smith JJ, Deane NG, Wu F, Merchant NB, Zhang B, Jiang A, et al. Experimentally derived metastasis gene expression profile predicts recurrence and death in patients with colon cancer. Gastroenterology. (2010) 138:95868. doi: 10.1053/j.gastro.2009.11.005

24. Laurent C, Valet F, Planque N, Silveri L, Maacha S, Anezo $\mathrm{O}$, et al. High PTP4A3 phosphatase expression correlates with metastatic risk in uveal melanoma patients. Cancer Res. (2011) 71:666-74. doi: 10.1158/0008-5472.CAN-10-0605

25. Kim HK, Choi IJ, Kim CG, Kim HS, Oshima A, Yamada Y, et al. Threegene predictor of clinical outcome for gastric cancer patients treated with chemotherapy. Pharmacogenom J. (2012) 12:119-27. doi: 10.1038/tpj.2010.87

26. Ooi $\mathrm{CH}$, Ivanova $\mathrm{T}, \mathrm{Wu}$ J, Lee $\mathrm{M}$, Tan IB, Tao J, et al. Oncogenic pathway combinations predict clinical prognosis in gastric cancer. PLoS Genetics. (2009) 5:e1000676. doi: 10.1371/journal.pgen.1000676

27. Forster S, Gretschel S, Jons T, Yashiro M, Kemmner W. THBS4, a novel stromal molecule of diffuse-type gastric adenocarcinomas, identified by transcriptome-wide expression profiling. Modern Pathol. (2011) 24:1390403. doi: 10.1038/modpathol.2011.99

28. Wang $\mathrm{G}, \mathrm{Hu} \mathrm{N}$, Yang $\mathrm{HH}$, Wang $\mathrm{L}$, Su $\mathrm{H}$, Wang $\mathrm{C}$, et al. Comparison of global gene expression of gastric cardia and noncardia cancers from a high-risk population in china. PLoS ONE. (2013) 8:e63826. doi: 10.1371/journal.pone.0063826

29. Brasacchio D, Busuttil RA, Noori T, Johnstone RW, Boussioutas A, Trapani JA. Down-regulation of a pro-apoptotic pathway regulated by PCAF/ADA3 in early stage gastric cancer. Cell Death Dis. (2018) 9:442. doi: 10.1038/s41419-018-0470-8

30. Cristescu R, Lee J, Nebozhyn M, Kim KM, Ting JC, Wong SS, et al. Molecular analysis of gastric cancer identifies subtypes associated with distinct clinical outcomes. Nat Med. (2015) 21:449-56. doi: 10.1038/nm.3850

31. Xie Y, Xiao G, Coombes KR, Behrens C, Solis LM, Raso G, et al. Robust gene expression signature from formalin-fixed paraffin-embedded samples predicts prognosis of non-small-cell lung cancer patients. Clin Cancer Res. (2011) 17:5705-14. doi: 10.1158/1078-0432.CCR-11-0196

32. Okayama H, Kohno T, Ishii Y, Shimada Y, Shiraishi K, Iwakawa $\mathrm{R}$, et al. Identification of genes upregulated in ALK-positive and EGFR/KRAS/ALK-negative lung adenocarcinomas. Cancer Res. (2012) 72:100-11. doi: 10.1158/0008-5472.CAN-11-1403

33. Huang P, Cheng CL, Chang YH, Liu CH, Hsu YC, Chen JS, et al. Molecular gene signature and prognosis of non-small cell lung cancer. Oncotarget. (2016) 7:51898-907. doi: 10.18632/oncotarget.10622

34. Tarca AL, Lauria M, Unger M, Bilal E, Boue S, Kumar Dey K, et al. Strengths and limitations of microarray-based phenotype prediction: lessons learned from the IMPROVER diagnostic signature challenge. Bioinformatics. (2013) 29:2892-9. doi: 10.1093/bioinformatics/btt492

35. Der SD, Sykes J, Pintilie M, Zhu CQ, Strumpf D, Liu N, et al. Validation of a histology-independent prognostic gene signature for early-stage, nonsmall-cell lung cancer including stage IA patients. J Thoracic Oncol. (2014) 9:59-64. doi: 10.1097/JTO.0000000000000042

36. Lee ES, Son DS, Kim SH, Lee J, Jo J, Han J, et al. Prediction of recurrencefree survival in postoperative non-small cell lung cancer patients by using an integrated model of clinical information and gene expression. Clin Cancer Res. (2008) 14:7397-404. doi: 10.1158/1078-0432.CCR-07-4937

37. Azimi F, Scolyer RA, Rumcheva P, Moncrieff M, Murali R, McCarthy SW, et al. Tumor-infiltrating lymphocyte grade is an independent predictor of sentinel lymph node status and survival in patients with cutaneous melanoma. J Clin Oncol. (2012) 30:2678-83. doi: 10.1200/JCO.2011.37.8539

38. Ohtani H. Focus on TILs: prognostic significance of tumor infiltrating lymphocytes in human colorectal cancer. Cancer Immun. (2007) 7:4.

39. Siegel RL, Miller KD, Jemal A. Cancer statistics, (2020). CA Cancer J Clin. (2020) 70:7-30. doi: 10.3322/caac.21590 
40. Muro K, Chung HC, Shankaran V, Geva R, Catenacci D, Gupta S, et al. Pembrolizumab for patients with PD-L1-positive advanced gastric cancer (KEYNOTE-012): a multicentre, open-label, phase 1b trial. Lancet Oncol. (2016) 17:717-26. doi: 10.1016/S1470-2045(16)00175-3

41. Le DT, Durham JN, Smith KN, Wang H, Bartlett BR, Aulakh LK, et al. Mismatch repair deficiency predicts response of solid tumors to PD-1 blockade. Science. (2017) 357:409-13. doi: 10.1126/science.aan6733

42. Du P, Xiong R, Li X, Jiang J. Immune regulation and antitumor effect of TIM-1. J Immunol Res. (2016) 2016:8605134. doi: 10.1155/2016/8605134

43. Zheng $\mathrm{X}, \mathrm{Xu} \mathrm{K}$, Chen $\mathrm{L}$, Zhou Y, Jiang J. Prognostic value of TIM1 expression in human non-small-cell lung cancer. J Transl Med. 17:178. doi: 10.1186/s12967-019-1931-2

44. Umetsu SE, Lee WL, McIntire JJ, Downey L, Sanjanwala B, Akbari O, et al. TIM-1 induces $\mathrm{T}$ cell activation and inhibits the development of peripheral tolerance. Nat Immunol. (2005) 6:447-54. doi: 10.1038/ni1186

45. Khademi M, Illes Z, Gielen AW, Marta M, Takazawa N, Baecher-Allan C, et al. T Cell Ig- and mucin-domain-containing molecule-3 (TIM-3) and TIM-1 molecules are differentially expressed on human Th1 and Th2 cells and in cerebrospinal fluid-derived mononuclear cells in multiple sclerosis. $J$ Immunol. (2004) 172:7169-76. doi: 10.4049/jimmunol.172.11.7169

46. de Souza AJ, Oriss TB, O’Malley K J, Ray A, Kane LP. T cell Ig and mucin 1 (TIM-1) is expressed on in vivo-activated $\mathrm{T}$ cells and provides a costimulatory signal for T cell activation. Proc Natl Acad Sci USA. (2005) 102:17113-8. doi: 10.1073/pnas.0508643102

47. Lee MJ, Woo MY, Chwae YJ, Kwon MH, Kim K, Park S. Downregulation of interleukin-2 production by CD4(+) T cells expressing TIM3 through suppression of NFAT dephosphorylation and AP-1 transcription. Immunobiology. (2012) 217:986-95. doi: 10.1016/j.imbio.2012.01.012

48. Mognol GP, Carneiro FR, Robbs BK, Faget DV, Viola JP. Cell cycle and apoptosis regulation by NFAT transcription factors: new roles for an old player. Cell Death Dis. (2016) 7:e2199. doi: 10.1038/cddis.2016.97

49. Wei X, Li H, Zhang Y, Li C, Li K, Ai K, et al. Ca(2+)-calcineurin axis-controlled NFAT nuclear translocation is crucial for optimal $T$ cell immunity in an early vertebrate. J Immunol. (2019) 204:56985. doi: 10.4049/jimmunol.1901065
50. Go CK, Hooper R, Aronson MR, Schultz B, Cangoz T, Nemani N, et al. The $\mathrm{Ca}(2+)$ export pump PMCA clears near-membrane $\mathrm{Ca}(2+)$ to facilitate store-operated $\mathrm{Ca}(2+)$ entry and NFAT activation. Sci Signal. (2019) 12:eaaw2627. doi: 10.1126/scisignal.aaw2627

51. Luo P, Wang L, Luo L, Wang L, Yang K, Shu G, et al. Ca(2+)-CalcineurinNFAT pathway mediates the effect of thymol on oxidative metabolism and fiber-type switch in skeletal muscle. Food Function. (2019) 10:516673. doi: 10.1039/C8FO02248H

52. Williams BL, Irvin BJ, Sutor SL, Chini CC, Yacyshyn E, Bubeck Wardenburg J, et al. Phosphorylation of Tyr319 in ZAP-70 is required for T-cell antigen receptor-dependent phospholipase C-gammal and Ras activation. EMBO J. (1999) 18:1832-44. doi: 10.1093/emboj/18.7.1832

53. Lin J, Chen L, Kane LP. Murine Tim-1 is excluded from the immunological synapse. F1000Research. (2012) 1:10. doi: 10.12688/f1000research. 1-10.v2

54. Encinas JA, Janssen EM, Weiner DB, Calarota SA, Nieto D, Moll T, et al. AntiT-cell Ig and mucin domain-containing protein 1 antibody decreases $\mathrm{TH} 2$ airway inflammation in a mouse model of asthma. J Allergy Clin Immunol. (2005) 116:1343-9. doi: 10.1016/j.jaci.2005.08.031

55. Umetsu DT, McIntire JJ, DeKruyff RH. TIM-1, hepatitis A virus and the hygiene theory of atopy: association of TIM1 with atopy. J Pediatric Gastroenterol Nutr. (2005) 40(Suppl. 1):S43. doi: 10.1097/00005176-200504001-00026

Conflict of Interest: The authors declare that the research was conducted in the absence of any commercial or financial relationships that could be construed as a potential conflict of interest.

Copyright (C) 2020 Kong, Fu, Niu and Jiang. This is an open-access article distributed under the terms of the Creative Commons Attribution License (CC BY). The use, distribution or reproduction in other forums is permitted, provided the original author(s) and the copyright owner(s) are credited and that the original publication in this journal is cited, in accordance with accepted academic practice. No use, distribution or reproduction is permitted which does not comply with these terms. 